\title{
Change Point Geometry for Change Detection in Surveillance Video
}

\author{
Brandon A. Mayer ${ }^{(凶)}$ and Joseph L. Mundy \\ Brown University, 182 Hope Street, Provdence, RI 02903, USA \\ brandon_mayer@brown.edu, mundy@lems.brown.edu
}

\begin{abstract}
A change detection algorithm is proposed based on geometric descriptors of space-time appearance discontinuities in fixed camera video. At each pixel in a video frame, intensity subsequences with similar appearance are segmented using a Hidden Semi-Markov Model (HSMM). The start of each per-pixel homogeneous subsequence, referred to as change point vertices, are then clustered across pixel locations using an efficient graph based segmentation algorithm to construct a change point hull. The geometry of the change point hull provides a discriminating feature for distinguishing coherent movement from random or stochastic appearance changes and is simultaneously a rich descriptor for reasoning about object velocity and direction. State of the art results are shown in change detection, a fundamental computer vision problem for identifying regions of video that exhibit meaningful variations as defined by the application context.
\end{abstract}

Keywords: Change detection $\cdot$ Video processing $\cdot$ Automated surveillance

\section{Introduction}

Change detection for fixed camera video sequences is a fundamental problem in computer vision which refers to a class of algorithms for detecting space-time regions in which a scene changes in a meaningful way. Change detection may be an end unto itself, e.g. as a monitoring tool in surveillance systems, or as a pre-processing step for further reasoning about regions identified as meaningful change. While change detection algorithms abound, these methods admit a simple three class taxonomy based on the semantic level of the underlying features of the approach: Pixel (low), Region (mid), and Object (high) level change detection algorithms.

Pixel or low level change detection algorithms attempt to classify meaningful changes on a per-pixel basis by distinguishing rare from common intensity sequences. While these algorithms tend to be computationally efficient, easy to parallelize and effective at identifying temporal discontinuities, they lack knowledge of the typical spatial structure of appearance variations and as such fail to reliably describe and distinguish between meaningful and irrelevant scene dynamics. Pixel level methods tend to trade simplicity for a shallow vocabulary

(C) Springer International Publishing Switzerland 2015

R.R. Paulsen and K.S. Pedersen (Eds.): SCIA 2015, LNCS 9127, pp. 377-387, 2015.

DOI: $10.1007 / 978-3-319-19665-7 \_31$ 


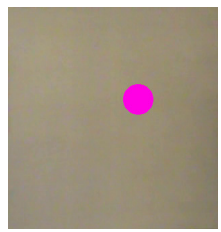

(a) Frame 0

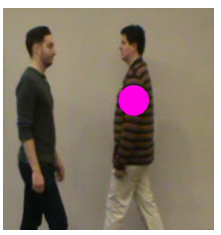

(b) Frame 130

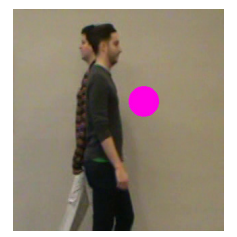

(c) Frame 140

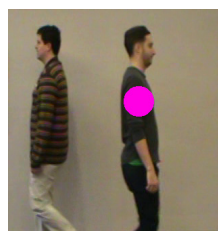

(d) Frame 155

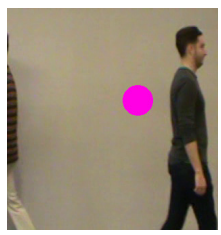

(e) frame 170

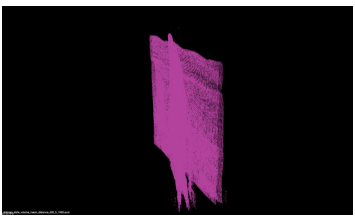

(f) Change-point vertex positions

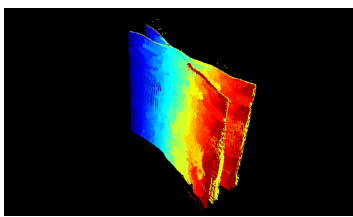

(g) Change-point hulls front view

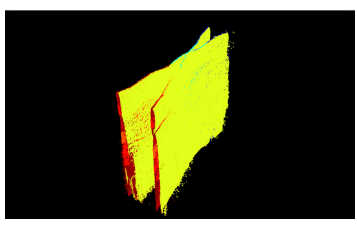

(h) Change-point hulls back view

Fig. 1. Crossing Paths sequence

with which to describe coherent action or behaviors, requiring significant ad-hoc post-processing that is typically algorithm specific and fragile.

Object (high) level change detection algorithms operate on the opposite end of the semantic spectrum, first attempting to recognize object categories present in a scene prior to reasoning about their behavior. While in theory this approach offers the most direct and rich vocabulary for describing scene dynamics, these systems suffer from severe practical limitations, requiring enormous amounts of training data per object category. For example, a generic object class such as a human may exhibit significant appearance variations under different lighting, camera resolutions or perspectives. Additionally, normalizing for interclass variability restricts the algorithms ability to transfer object models to different applications and scenes. Further compounding the problem, it is impossible to define apriori all object classes which may be observed in a video sequence, let alone learning or building an accurate object model for each class.

Region (mid) level change detection algorithms identify space-time features that are correlated with object behavior without explicitly localizing and classifying all object classes in a scene. The proposed region or mid level method is a compromise between the efficient low-level and descriptive but impractical high-level approaches which uses the geometry of space-time discontinuities to localize and quantify coherent actions in video sequences.

First, a Hidden Semi-Markov Model (HSMM) is used to segment per-pixel observations into temporal regions with similar appearance and duration. At each pixel, the start of a segmented intensity subsequence, a $3 \mathrm{D}$ point in $2 \mathrm{D}$ space-time known as a change-point vertex, marks the beginning of an approximately homogeneous intensity sequence which differs significantly from the previous segment [10]. An efficient graph-based clustering algorithm [4] is used to group neighboring change-point vertices based on the expected appearance of the temporal segment to form a change-point hull. The change-point hull could 
be thought of as space-time "super-voxel" of discontinuities, demarcating regions of dissimilar appearance. The initial segmentation retains the efficiency of pixel level algorithms while the clustering step builds a rich, data-driven space-time descriptor with minimal computational overhead. The shape of the changepoint hulls (clusters) provide a discriminating vocabulary for describing coherent motion in video sequences.

As a motivating example, consider the "Crossing Paths" sequence of fig. 1. In this video, a camera has been observing an indoor area when two people walk into the scene, crossing paths approximately at the frame center, and continue past the camera view. First, the per-pixel algorithm groups similar appearances into segments along the temporal axis as visualized in fig. 4a which shows a single pixel intensity sequence stacked as a vertical column. The initial temporal segmentation corresponding to the pixel location at the center of the pink circle would produce temporal groups that would be correlated with the semantic class sequence: (wall appearance, person one appearance, wall appearance, person two appearance, wall appearance). The beginning of each temporal segment defines a point in 3D (2D space-time) called a change point vertex. Figure $4 \mathrm{~b}$ visualizes change point vertices, the image space-time location of temporal appearance discontinuities, as black spheres. The edges that are constructed between neighboring change point vertices in a later step are visualized as dotted lines. Figure if shows the location of all change point vertices recovered after the pixel independent segmentation for the "Crossing Paths" video as points in video frame space-time.

Weighted edges are constructed between neighboring change vertices and clustered using a graph based segmentation algorithm [4] where each cluster of change point vertices are referred to as change point hulls. Figures $1 \mathrm{~g}$ and $1 \mathrm{~h}$ show the recovered change-point hulls visualized as points in space time where color denotes hull membership. While there may be appearance variations within a single object class (the person walking from right to left has a multi-colored shirt, beige pants and dark hair), the change point hulls exhibit a coherent planar shape. Figure 1f shows the change point vertices as the people occlude the wall. The wall appearance is so uniform that in figure $1 \mathrm{~h}$ the transition from each person back to the wall is all assigned to the same change hull (yellow).

As shown in the "Crossing Paths" experiment, coherent object motion induces planar geometries with respect to the change point hulls while the velocity of object motion is correlated with the normal direction of the change-point hull with respect to the principle axis of the camera. Furthermore, by observing the relative positions in space and time of change point hulls, it is possible to reason about object trajectory, velocity and occlusion while further experiments reveal image noise, random and incoherent motion induce elliptical or spherical change-point surfaces.

An example of a more complex change hull geometry generated by periodic motion is shown in fig. 2c. The wind turbine moves in a periodic circular motion which pixel-level change detection algorithms struggle to model as background motion [10]. However, the recovered change point hulls exhibit a coherent 
structure with a global helical shape that can be approximated locally with connected planes.

The singular values of a 3D spatial covariance matrix composed of the location of change point vertices within the same change point hull (cluster) is used to quantify the "planarity" of the space-time discontinuity for identifying coherent motion and recognizing meaningful scene changes.

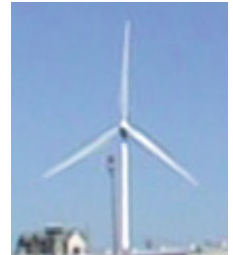

(a) Frame 0

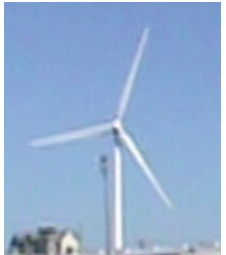

(b) Frame 8

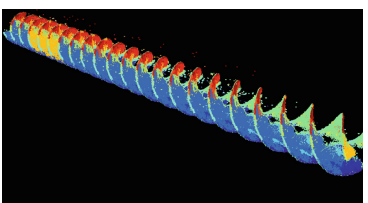

(c) Change Hulls

Fig. 2. Fields Point Turbine

\section{Related Work}

Pixel level methods are the most common class of algorithms for change detection [1,2,6-8, 12,14]. Gaussian Mixture Models [7,14] are a standard parametric per-pixel intensity model where each pixel location in video is modeled using a mixture of Gaussians distribution. Many heuristics have been proposed for associating certain modes of the mixture distribution with the background or foreground of a scene [14], removing shadows and increasing parametric learning accuracy and speed [7], and incorporating multiple models of slowly occurring or fast intensity changes as well as other region-level edge statistics [2]. However, each of these methods suffer from the same problems in that a GMM can only model typical pixel intensities, the order and duration of a pixel appearance has no effect on the probability of the observed pixel intensity sequence.

Low-level change detection methods also include non-parametric models such as $[1,6]$ which explicitly store a history of observed intensities at each pixel. Both algorithms specify routines where per-pixel background models "forget" about unlikely past observations and update the history with more common intensities by randomly resampling the memory buffer. Again these models suffer from permutation invariance; shuffling frames of video does not produce significantly different background models. Additionally, the amount of memory assigned to each pixel's memory buffer implicitly defines an upper bound for modeling periodic phenomena such as the turbine of fig. 2 .

The method described in [12] transforms a windowed history of intensity observations to the frequency domain to associate common Fourier coefficients with background at each pixel in a video sequence and a meaningful change 
as a significant deviation from an average of previously computed frequency coefficients. While this method does indirectly model intensity sequences, the parameter governing the window length implicitly sets a fixed scale on the type of temporal patterns the algorithm can reliably model and, like other low level methods, must define algorithm specific heuristics to account for spatially coherent motion and behaviors.

The duration dependent codebook proposed in [10] models intensity sequences at every pixel location in a frame of video using a Duration Dependent Markov Model (DDHMM). During a training phase, a codebook is constructed based on local state assignments, mappings between per-pixel intensity subsequences and DDHMM state-duration pairs that summarize the appearance and temporal statistics of each intensity segment. State-duration assignments that occur during new video of the same scene that are uncommon relative to the training codebook are labeled as significant change. While the proposed method uses the same per-pixel DDHMM model and learning algorithm proposed in [10], this research investigates the plausibility of analyzing the geometry of clustered per-pixel discontinuities for visual reasoning and change detection. The novel contributions of this work relative to [10] include the introduction of change point vertices, the position of a state label transition in space-time representing a pixel-independent discontinuity. Change point vertices are clustered to form change point hulls that represent local patches of appearance discontinuities that transition to similar intensities. Finally, a novel classifier based on shape descriptors of change point hulls, derived via spectral analysis of scatter matrices composed of the space-time positions of vertices associated with each change point hull is used to detect changes in video.

The Spatial-Temporal Local Binary Pattern change detection algorithm [15] (STLBP) uses a local gradient descriptor for identifying common textures in video. While the STLBP descriptor is the most similar feature of the reviewed algorithms to the proposed method of analyzing spatial-temporal discontinuities there are major differences. First, the STLBP algorithm defines a fixed local neighborhood for computing the gradient around each pixel while the proposed algorithm uses a data driven approach to directly model the natural scale of homogeneous sequences and their corresponding discontinuities. Secondly, STLBP describes local texture using a gradient histogram, taking a bag-of-words approach. The shape of the appearance discontinuities is therefore lost, and with it the ability to reason about higher level motion or behaviors occurring in target videos. The proposed method however, uses the shape of appearance discontinuities as a fundamental feature for local scene description and change detection.

The work of [11] attempts to detect the presence of predefined activities in video such as "watching TV" or "making tea" using object recognition. This high level algorithm required assembling a dataset of over 1 million frames of video for learning only 18 activities. The authors acknowledge inter-class appearance variability as a source of significant difficulties, citing the example that a refrigerator looks very different when it is closed compared to when it is opened. Standard unsolved object recognition challenges and benchmarks such as the 
PASCAL challenge [3] consist of almost 12,000 images for training and evaluating classification of 20 object categories while the ambitious ImageNet [13] consists of over 14 million annotated images and 10,000 object classes. These datasets and challenges underscore the difficulty in modeling the appearance variability of even a small number of pre-defined object classes even in an era of ubiquitous computing and big-data. Because robust object detection is still an unsolved computer vision task, it is necessary for performant, state of the art change detection algorithms to operate within the low or medium levels of the change detection semantic taxonomy, at least for the foreseeable future.

\section{Algorithm}

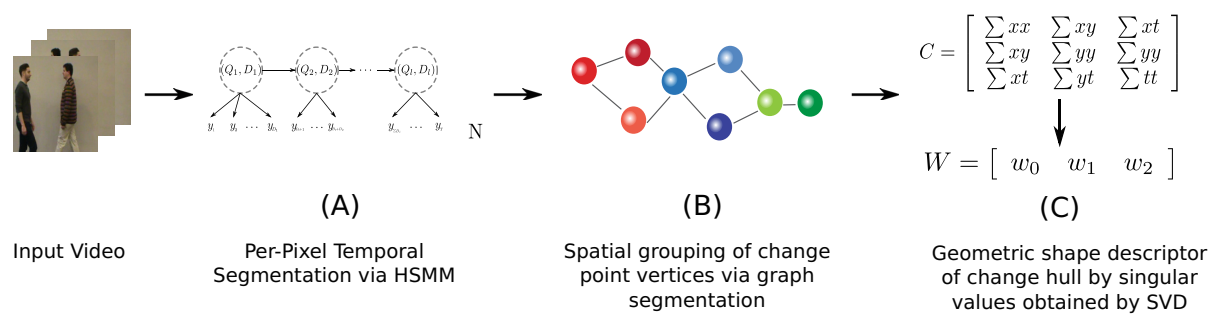

Fig. 3. Visualization of proposed algorithm. Each pixel of an input video is segmented into intensity subsequences with similar appearance and duration using a Hidden SemiMarkov Model. The beginning of each subsequence, a 3D point in 2D space-time and known as a change point vertex, are clustered into groups with similar appearance using a graph segmentation algorithm to form a change point hull. The vertices within a change point hull are used to construct a spatial covariance matrix which is decomposed via Singular Value Decomposition (SVD) to obtain the eigenvector and eigenvalues of the changepoint hull. The eigenvalues are then used as a descriptor for coherent motion and detecting meaningful appearance aberrations.

An outline of the proposed method is shown graphically in fig. 3. The intensity time series associated with each pixel location of fixed camera video is modeled using a class of Hidden Semi-Markov Models [5,9] known as a Duration Dependent Markov Model (DDHMM). The learning algorithm outlined in [10] is applied to each pixel location independently to produce an initial per-pixel segmentation of approximately homogeneous appearance subsequences. Temporal appearance discontinuities are then clustered using the graph-based segmentation algorithm described in [4] to group similar neighboring appearance discontinuities. The eigenvalues of the spatial covariance matrix composed of the locations of appearance discontinuities within the same cluster are used as a shape descriptor for identifying coherent motion and detecting meaningful changes. 

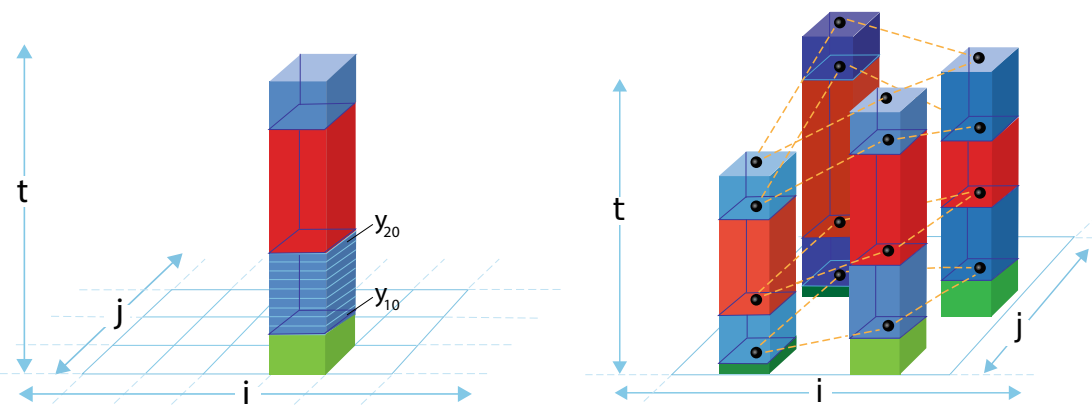

(a) Initial pixel independent temporal(b) Clustering per-pixel change vertices via appearance segmentation. graph segmentation

Fig. 4. Figure 4a shows the independent per-pixel temporal segmentation of intensity subsequences into homogeneous groups with similar appearance and duration. The beginning of each sequence, known change point vertices, are visualized in figure $4 \mathrm{~b}$ as black spherical points in $3 \mathrm{D}$ (2D image space and time). Each change point vertex is connected to its four nearest neighbors and assigned an edge weight which is used for segmenting the change hull.

\subsection{Per-Pixel Intensity Sequence Model}

An input video sequence is decomposed into a collection of per-pixel intensity time series: $Y(i, j)=\left(y_{1}, \ldots, y_{T}\right)$, one for each pixel location $(i, j)$ for a video sequence with $T$ frames. Denoting a random variable with a capital letter and specific instantiations of the corresponding variable with the matching lower case, a DDHMM models the sequence of observations using a sequence of latent state pairs: $\left.\left(S_{1}, D_{1}\right),\left(S_{2}, D_{2}\right), \ldots,\left(S_{l}, D_{l}\right)\right)$ where $S_{i}$ is a state label and $D_{i}$ is a random variable that represents the time spent in state $S_{i}$ [10].

The DDHMM joint probability of a per-pixel intensity sequence and stateduration sequences is computed according to

$$
\begin{gathered}
p\left(y_{1}, \ldots, y_{T}, s_{1}, d_{1}, \ldots, s_{L}, d_{L}\right)=\underbrace{p\left(s_{1}\right) p\left(d_{1} \mid s_{1}\right) \prod_{i=1}^{d_{1}} p\left(y_{i} \mid s_{1}\right) \cdots}_{\text {Initial state-duration }} \\
\underbrace{\prod_{l=2}^{L-1} p\left(d_{l} \mid s_{l}\right) p\left(s_{l} \mid s_{l-1}\right) \prod_{j=1}^{d_{l}} p\left(y_{r_{i}+j} \mid s_{l}\right)}_{\text {Internal state-durations, transitions and emission }} \underbrace{p\left(D_{L} \geq d_{L}\right) p\left(s_{L} \mid s_{(L-1)}\right) \prod_{z=1}^{d_{L}} p\left(y_{r_{(L-1)}+z} \mid s_{L}\right)}_{\text {right-censor term }}
\end{gathered}
$$

Where $p\left(D_{i}=d_{i} \mid S_{i}=s_{i}\right), p\left(S_{i}=s_{i} \mid S_{i-1}=s_{i-1}\right)$ and $p\left(y_{t} \mid S_{i}=s_{i}\right)$ are the duration, state transition and emission distributions respectively, $r_{i}=\sum_{m=1}^{i} d_{m}$ and $p\left(s_{1}\right)$ is an initial distribution of state labels. The term $p\left(D_{l} \geq d_{l} \mid s_{l}\right)$ is called the state survival distribution and is included in the computation to account for the fact that 
the end of an observation sequence may not coincide with the end of a homogeneous subsequence and may just be a consequence of missing data [5]. The duration and transition distributions were chosen to be multinomial with zero self-transition probability while the emission distributions are univariate Gaussian. A graphical representation of the likelihood function is shown in standard graph-plate notation in step (A) of fig. 3 and indicates there is one HSMM for each of the $N$ pixels in each frame of video.

\subsection{Temporal Appearance Discontinuities}

The computationally efficient algorithm developed in [10] is employed for learning the parameters and complexity of the per-pixel DDHMM model. The algorithm processes each intensity observation $y_{t}$ in order, testing three hypothesis by comparing the current observation with the existing state conditional distributions: Extend the current state-duration label $\left(s_{l}, d_{l}\right)$ by associating observation $y_{t}$ with the current segment, associating $y_{t}$ with an already existing state, or creating a new state label. Model complexity is regularized using a modified $A I C$ criteria and the reader is referred to [10] for the remaining details.

The output of the per-pixel learning algorithm are the parameters of the emission, transition and duration distributions and assignments of pixel observations $\left(y_{1}, \ldots, y_{t}\right)$ to state-duration segments: $\left(\left(s_{1}, d_{1}\right), \ldots,\left(s_{l}, d_{l}\right)\right)$.

\subsection{Clustering Change Point Vertices}

The state duration pair assignments $\left(\left(s_{1}, d_{1}\right), \ldots,\left(s_{l}, d_{l}\right)\right)$ run-length encode temporal appearance discontinuities within an observation sequence. For any given pixel location $(i, j)$, the temporal location of the $k^{t h}$ discontinuity can be computed as $t_{k}=\sum_{m=1}^{k-1} d_{m}$, recovering a vertex in three dimensions representing a local temporal appearance discontinuity at video frame location $\left(i, j, t_{k}\right)$.

Each local temporal discontinuity (change point vertex) is connected to its four nearest neighbors as shown in fig. $4 \mathrm{~b}$. Figure $4 \mathrm{~b}$ visualizes per-pixel intensity sequences from video as vertical columns. The beginning of semi-homogeneous temporal subsegments, the change point vertices, are shown as black spheres. Edges are created that connect a given change point vertex to its closest neighbor in each of its four nearest columns (pixel sequences). Each edge is weighted by the absolute difference in expected value of the state conditional emission distribution for the state assigned to the respective change vertex by the learning algorithm in 3.2. The graph-based clustering algorithm described in [4] is used to spatially group per-pixel temporal discontinuities based on similar appearance. While any spatially aware clustering method could be used, the algorithm in [4] is not only extremely fast in practice, easily scaling to millions of change point vertices, but has favorable theoretical complexity guarantees and is a standard tool in the computer vision community. The graph segmentation algorithm defines a spatial scale parameter which is set to 300 in all experiments.

\section{Detecting Change}

A background model is created by first dividing the image plane into a non-overlapping grid of cells. During a training phase, each change point hull is assigned to a grid cell according to the majority membership of its vertices. First, a threshold is applied to 
the smallest eigenvalue of the change point hull. If the smallest eignvalue is greater than $\tau_{w}$, it is assumed the hull does not fit a planar model and thus does not represent coherent motion and is discarded. For all remaining change point hulls, the angle of the normal direction of the hull is computed with respect to the reference by projecting the smallest eigenvector of the data covariance matrix decomposition onto the image plane. For each cell of the grid, a $2 \mathrm{~d}$ histogram of normal directions and absolute value of the smallest eigenvalue, a measure of how well a planar model fits the change point hull, is maintained as a background model.

During testing, the eignenvalue threshold $\tau_{w}$ is again applied to each change hull, discarding hulls which do not fit a planar model. The remaining change hulls are assigned to grid cells and compared with the background histogram. Deviations, from the background model are determined by thresholding the joint probability of a change hull's normal direction and smallest singular value with respect to the background model by a constant $\tau_{h}$. All vertices that comprise a hull deemed to be abnormal are labeled as change. In all experiments, the grid cell size was set to $20 \times 20$ pixels, $\tau_{w}=0.3$ and $\tau_{h}=0.2$ and empirically determined.

\section{Empirical Results and Discussion}

Figures 5 and 6 visualize the results of the proposed change detection system. In figure 5, a person walks into the scene from right to left, and most of the person is detected as change. However, his pants are a very similar color to the hallway rendering it difficult to detect appearance discontinuities between the pants and the background resulting in false negatives on the pedestrians legs.

Figure 6 shows a different pedestrian walking into view, stopping in the center before continuing off frame. Again, most pixels associated with the pedestrian are
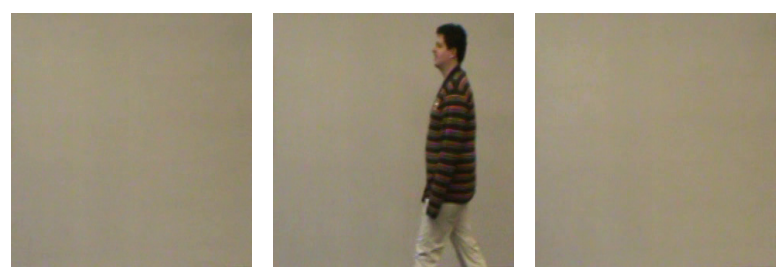

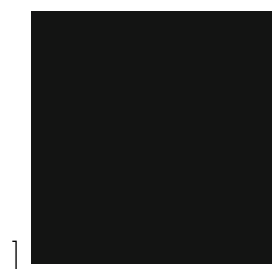

(a) Frame 0

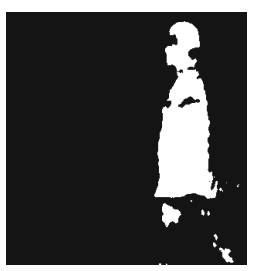

(b) Frame 134

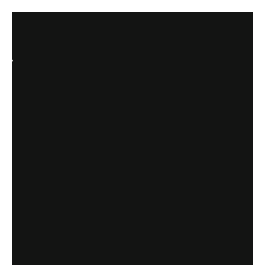

(c) Frame 195

Fig. 5. Detecting changes in a scene monitoring a hallway. The top row are the original frames of video, the bottom visualizes changes detected by the algorithm as white pixels and black as background. 

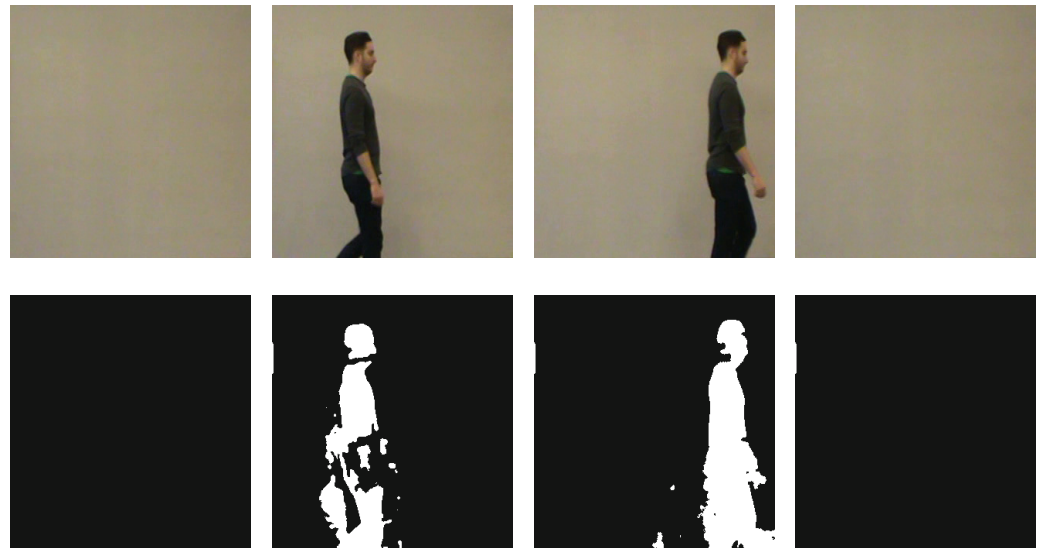

Fig. 6. Change detection results for person walking into a scene, stopping, then continuing forward. The top row are the original frames and the bottom visualizes detected changes as white and background is colored black.

detected, however there are a few errors owing to the similar color of the background to the pedestrians skin color and shadows.

The proposed change point vertices and hulls show promise as effective features for change detection. Future work will focus on coupling the planar model and change point hull clustering algorithm for directly segmenting locally planar spatio-temporal appearance discontinuities rather than the bottom up approach presented. Modules for detecting and removing shadows will also be incorporated to recover a more refined segmentation. Additionally, it is desirable that future work relax the fixed camera assumptions in order to extend the applicability of the described approach as more ego-centric and multi-view datasets have become commonplace.

\section{References}

1. Barnich, O., Van Droogenbroeck, M.: Vibe: A universal background subtraction algorithm for video sequences. IEEE Transactions on Image Processing 20(6), 1709-1724 (2011). doi:10.1109/TIP.2010.2101613

2. Evangelio, R.H., Paetzold, M., Keller, I., Sikora, T.: Adaptively splitted gmm with feedback improvement for the task of background subtraction. IEEE TRANSACTIONS on Information Forensics and Security (accepted for publication)

3. Everingham, M., Van Gool, L., Williams, C., Winn, J., Zisserman, A.: The pascal visual object classes (voc) challenge. International Journal of Computer Vision 88(2), 303-338 (2010). doi:10.1007/s11263-009-0275-4. http://dx.doi.org/10.1007/s11263-009-0275-4

4. Felzenszwalb, P.F., Huttenlocher, D.P.: Efficient graph-based image segmentation. International Journal of Computer Vision 59 (2004)

5. Guédon, Y.: Estimating hidden semi-markov chains from discrete sequences. Journal of Computational and Graphical Statistics 12(3), 604-639 (2003). http://www.jstor.org/stable/1391041 
6. Hofmann, M., Tiefenbacher, P., Rigoll, G.: Background segmentation with feedback: the pixel-based adaptive segmenter. In: 2012 IEEE Computer Society Conference on Computer Vision and Pattern Recognition Workshops, CVPRW, pp. 38-43 (2012). doi:10.1109/CVPRW.2012.6238925

7. Kaewtrakulpong, P., Bowden, R.: An improved adaptive background mixture model for real-time tracking with shadow detection. In: Proceedings of 2nd European Workshop on Advanced Video Based Surveillance Systems, vol. 5308 (2001)

8. Kim, K., Chalidabhongse, T.H., Harwood, D., Davis, L.S.: Real-time foregroundbackground segmentation using codebook model. Real-time Imaging 11, 172-185 (2005). doi:10.1016/j.rti.2004.12.004

9. Levinson, S.: Continuously variable duration hidden markov models for automatic speech recognition. Computer Speech \& Language 1(1), 29-45 (1986). doi:10. 1016/S0885-2308(86)80009-2. http://www.sciencedirect.com/science/article/pii/ S0885230886800092

10. Mayer, B.A., Mundy, J.L.: Duration dependent codebooks for change detection. In: Proceedings of the British Machine Vision Conference, BMVC (2014)

11. Pirsiavash, H., Ramanan, D.: Detecting activities of daily living in first-person camera views. In: 2012 IEEE Conference on Computer Vision and Pattern Recognition (CVPR). IEEE (2012)

12. Porikli, F., Wren, C.: Change detection by frequency decomposition: wave-back. In: Proc. of Workshop on Image Analysis for Multimedia Interactive Services (2005)

13. Russakovsky, O., Deng, J., Su, H., Krause, J., Satheesh, S., Ma, S., Huang, Z., Karpathy, A., Khosla, A., Bernstein, M., Berg, A.C., Fei-Fei, L.: ImageNet Large Scale Visual Recognition Challenge (2014). arXiv:1409.0575

14. Stauffer, C., Grimson, W.E.L.: Adaptive background mixture modelsfor real-time tracking. In: IEEE Computer Society Conference on Computer Vision and Pattern Recognition, vol. 2, p. 252 (1999). doi:10.1109/CVPR.1999.784637

15. Zhang, S., Yao, H., Liu, S.: Dynamic background modeling and subtraction using spatio-temporal local binary patterns. In: 15th IEEE International Conference on Image Processing, ICIP 2008, pp. 1556-1559 (2008). doi:10.1109/ICIP.2008. 4712065 